\title{
BMJ Open Chronic non-bacterial osteitis from the patient perspective: a health services research through data collected from patient conferences
}

\author{
Colen Cooper Gore Silier, ${ }^{1}$ Justina Greschik, ${ }^{2}$ Susanne Gesell, ${ }^{2}$ Veit Grote, ${ }^{1}$ \\ Annette F Jansson ${ }^{1}$
}

To cite: Silier CCG, Greschik J, Gesell S, et al. Chronic nonbacterial osteitis from the patient perspective: a health services research through data collected from patient conferences. BMJ Open 2017;7:e017599. doi:10.1136/ bmjopen-2017-017599

- Prepublication history for this paper is available online. To view these files, please visit the journal online (http://dx.doi org/10.1136/bmjopen-2017017599).

Received 9 May 2017 Revised 8 September 2017 Accepted 16 October 2017

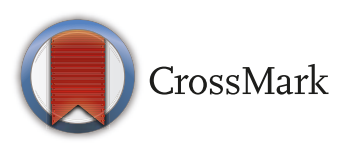

${ }^{1}$ Department of Rheumatology and Immunology, Dr. von Hauner Children's Hospital, Ludwig-

Maximilians-University, Munich, Germany

${ }^{2}$ Department of Orthopaedic Surgery, Physical Medicine and Rehabilitation, Ludwig-

Maximilians-University, Munich, Germany

\section{Correspondence to} Mrs. Colen Cooper Gore Silier; colen.silier@campus.Imu.de, colen.silier@gmail.com

\section{ABSTRACT}

Objective Although chronic non-bacterial osteitis (CNO) is an ever-increasingly recognised illness in the paediatric community and the adult healthcare community, a study to assess diagnosing, treatment and the psychosocial aspect of CNO from a large population pool was not available. We aimed to investigate CNO from the patient perspective. Design Health services research, patient survey. Setting Ludwig-Maximilians-University (LMU) Pediatric Rheumatology Department CNO Conferences held in June 2013 and June 2015.

Participants Using a patient survey developed by the LMU Pediatric Rheumatology Department, 105 patients from ages 5 to 63 years were assessed regarding CNO to include epidemiological data, medical history and treatment, initial symptoms, diagnostic procedures, current symptoms, associated diseases, current treating physicians, absences in school and work due to illness and the impact of illness on patient, family and friends.

Results Active CNO was reported in $90 \%$ of patients present, with $73 \%$ being women and $27 \%$ being men. An overwhelming majority $(70 \%)$ reported being diagnosed within 18 months of onset of symptoms; however, the initial diagnoses were wide-ranged to include malignancies in $36 \%$ to bacterial osteomyelitis in $30 \%$, where the majority were treated with an antibiotic and/or were biopsied. When asked about the psychosocial aspect of this illness, $83 \%$ reported that non-bacterial osteitis (NB0) negatively impacted the family, $79 \%$ reported that NBO has negatively affected either school or work and $56 \%$ reported a negative impact on friendships.

Conclusion Delay of diagnosis, living with differential diagnoses like malignancies and finding specialists for medical care are a few examples of what leads patients into searching for more information. The negative impact on daily life including family relationships, friendships and work/school highlights a need for better psychosocial support such as guidance counselling or psychological support due to three-quarters of patients receiving no such said support.

\section{INTRODUCTION}

Osteomyelitis is often assumed to be of bacterial origin even in the absence of a pathogen; however, current research supposes that a

\section{Strengths and limitations of this study}

- This is the first study highlighting the impact of chronic non-bacterial osteitis (CNO) from the patient perspective.

- A relatively large patient population for CNO was analysed, which has an incidence rate of $0.45 / 100000$.

- The explicitness of the needs of patients with CNO was examined, while stressing the psychosocial and socioeconomic effect of a chronic illness, such as CNO.

- The patient data reflect the current medical literature concerning $\mathrm{CNO}$, therefore further validating the patient information gathered.

- A major limitation lies in the retrospective analysis of different time frames required by our study's participants.

leading portion of non-bacterial bone lesions are of an autoinflammatory origin. Furthermore, due to the ever-increasing use of MRI, bone lesions are increasingly being found in healthy children and adults alike. ${ }^{12}$

Non-bacterial osteitis (NBO) can affect one bone or more often, multiple bones; therefore, it is often best known by its most severe manifestation chronic recurrent multifocal osteomyelitis (CRMO) (figure 1) with a multifocal sterile osteitis. ${ }^{3-9}$ The chief complaint of localised bone pain often results in identifying multifocal or unifocal lesions which can appear in all skeletal sites ${ }^{3-7}$ and progression can vary widely to include acute, chronic persistent or chronic relapsing. ${ }^{6}$

Because chronic non-bacterial osteitis $(\mathrm{CNO})$ is a chronic illness, it was important to be able to assess the psychological and social impact on patients throughout the illness. Maslow et al studied chronically ill children in regards to social, educational and vocational outcomes, coming to the conclusion that socially, the paediatric population studied was 


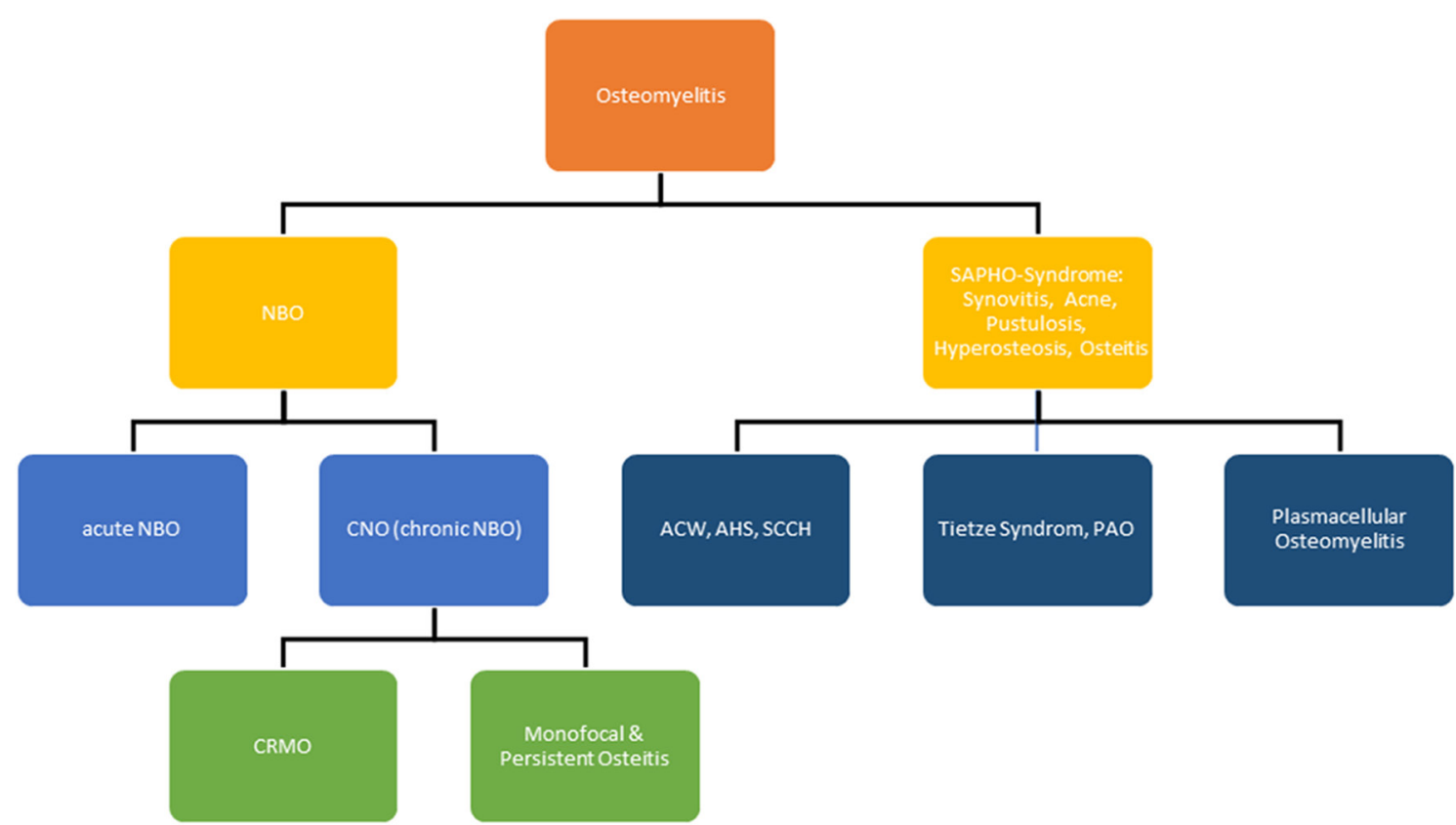

Figure 1 Terminology of NBO. ACW, Anterior Chest Wall Syndrome; AHS, Acquired Hyperostosis Syndrome; CRMO, chronic recurrent multifocal osteomyelitis; NBO, non-bacterial osteitis; PAO, Pustulotic arthro-osteitis; $\mathrm{SCCH}$, Sternocostoclavicular Hyperostosis.

not discriminated against, but they did have more difficulty with educational and vocational opportunities. ${ }^{10}$ Chronic illness, however, does affect the patient and the family and support structure; it has been suggested that the adaptation of the patient and the family is closely linked. ${ }^{11} 12$

We assessed patients with diagnosed CNO using a questionnaire that was developed to encompass the onset of symptoms to diagnostics and then on to the social aspect of the chronically ill and access to care issues. Specifically, how well is the patient informed about $\mathrm{CNO}$ and what does the patient require (information-wise and other needs) were addressed, with emphasis on the psychosocial aspects.

\section{METHODS}

\section{Study design and study population}

In June 2013 and June 2015, the Pediatric Rheumatology Department of the Ludwig-Maximilians-University Munich hosted an NBO information day designed for patients, both paediatric and adults, and their relatives. The event was advertised through private practice paediatricians, private practice rheumatologists, websites dedicated to paediatric rheumatology and university clinics throughout Germany. Patients and their families were asked to register 2 weeks in advance, and on registration they received a survey and a consent form to be filled out and brought with to the conference.

In total, 134 patients were in attendance, with 107 patients completing the survey. In June 2013, 69 patient surveys were collected, and 38 were collected in 2015. Patients were asked to not fill out a survey in 2015 if they had previously done so in 2013. There were 13 patients which visited both conference days, and therefore did not repeat the survey. However, 14 patients did not respond due to appearing without prior registration or registering after the 2-week deadline.

The patient survey consisted of 285 variables/patient and captured important aspects of NBO to include: epidemiological data, age at diagnosis, family history, medical and treatment history, constitutional symptoms at disease onset, diagnostic procedures, number of lesions and associated diseases in patients and in family members (parents and siblings).

The survey also focused on: who is the consulting physician, how far away is the specialist, physical therapy options and absences in school or at work due to disease. The psychosocial impact concentrated on the impact of the illness on the patient, friends and family.

We specifically asked in our survey about three initial symptoms: pain, swelling and redness, and pain was rated on a Visual Analogue Scale (VAS) of 1-10, with 10 being maximum pain.

\section{Statistical analysis}

All data management and analysis were performed using IBM SPSS Statistics V.23. Continuous variables were expressed in means with SD or-if skewed-as medians with IQRs (IQR: 25th-75th percentiles). The Student's t-test was used to compare quantitative data with $\mathrm{P}$ values below 0.05 considered to be statistically significant. The Pearson's $\chi^{2}$ test was used for differences of categorical data.

\section{RESULTS}

General

During the 2-year survey period, we received a total of 107 surveys, of these questionnaires, two were incomplete and could not be used for further analysis. Overall, data were 


\section{First diagnoses}

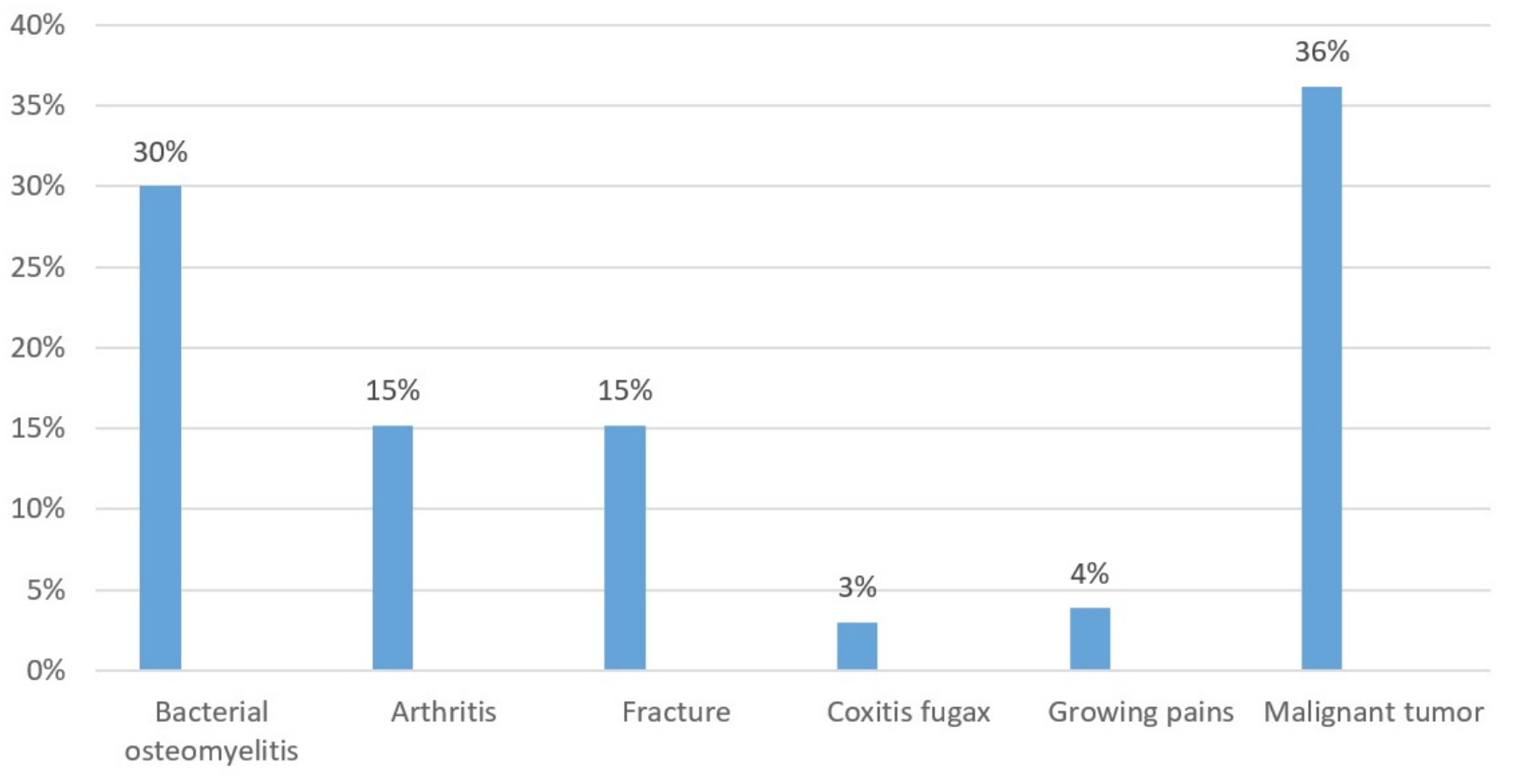

Figure 2 First diagnoses.

collected from 105 patients, 67 from the 2013 conference and 38 from 2015. Active CNO was reported in $90 \%$ $(\mathrm{n}=94)$ of patients present.

\section{Epidemiology}

From 105 patients, $73 \%(\mathrm{n}=77)$ were female and $27 \%$ $(n=28)$ were male. A total of $18 \%$ of the patients living in Germany have a non-German parent $(3 \%)$ or both parents are of non-German nationality (15\%). Eight international patients were also present, residing in other European countries such as Switzerland, Austria and Sweden. Ages of this collective ranged from 5.5 years to 63 years, with an average of age of 16.7 years (SD 8.5). Thirty-two patients $(30.5 \%)$ were $>18$ years old.

Symptom onset occurred at a median of 9.5 years of age (IQR: 7.5-12), and the median age at the time of diagnosis was 10.5 years (IQR: $8.5-13.5$ ), with $86 \%$ reporting onset of symptoms between the ages of 6 and 15 years.

\section{Clinical presentation}

Our patients were initially referred to a variety of physicians including paediatricians, general practitioners, orthopaedic surgeons, rheumatologists (both paediatric and adult), oral and maxillofacial surgeons, dermatologists and ear-nose-throat physicians. The most common first diagnoses are shown in figure 2, with some receiving multiple first diagnoses. Under malignant tumours/ malignant disease, patients listed-unknown: $18 \%$, Ewing's sarcoma: 6\%, leukaemia: 3\% and Langerhans cell histiocytosis: $2 \%$.
Paediatric rheumatologists diagnosed in $57 \%$ of the CNO cases present. Overall rheumatologists and paediatricians made the diagnosis in $69 \%$ of all patients. Only $6 \%$ were diagnosed after consultation with one physician, and $69 \%$ consulted with 2-5 physicians before receiving the final diagnosis. One patient was referred to a total of 15 different physicians before receiving the diagnosis of CNO.

At the time of survey, the median length of CNO symptoms was 3.92 years (IQR: 1.83-6.83), and the median length from the time of diagnosis was 2.17 years (IQR: 0.92-5.08).

Pain was reported as the number one initial symptom $(97 \%)$, followed by swelling at $60 \%$ and redness at $25 \%$. Fever of unknown origin was reported in $17 \%$. An overwhelming majority of patients $(65 \%)$ reported being in constant pain at the start of this syndrome with peak-pain times being in the evening $(36 \%)$. At initial presentation, $20 \%$ rated pain on a VAS (0-10) as an $8,23 \%$ at a 9 and $23 \%$ at a 10 . Patients rated current pain levels to be significantly lower; approximately $55 \%$ of patients rated pain to be a 4 or below and $81 \%$ as a 6 or below.

Former or current elevated inflammation parameters $(\mathrm{C}$ reactive protein $(\mathrm{CRP})$ and erythrocyte sedimentation rate (ESR)) were reported in $45 \%$ of patients.

A precipitating event or illness is believed to be the cause of CNO in $45 \% \quad(n=47)$ of patients. From the 47 patients, $14(30 \%)$ believe this trigger to be a bacterial infection and 9 (19\%) believe this to be viral. A trauma, which was directly related to the emergence of $\mathrm{CNO}$, 


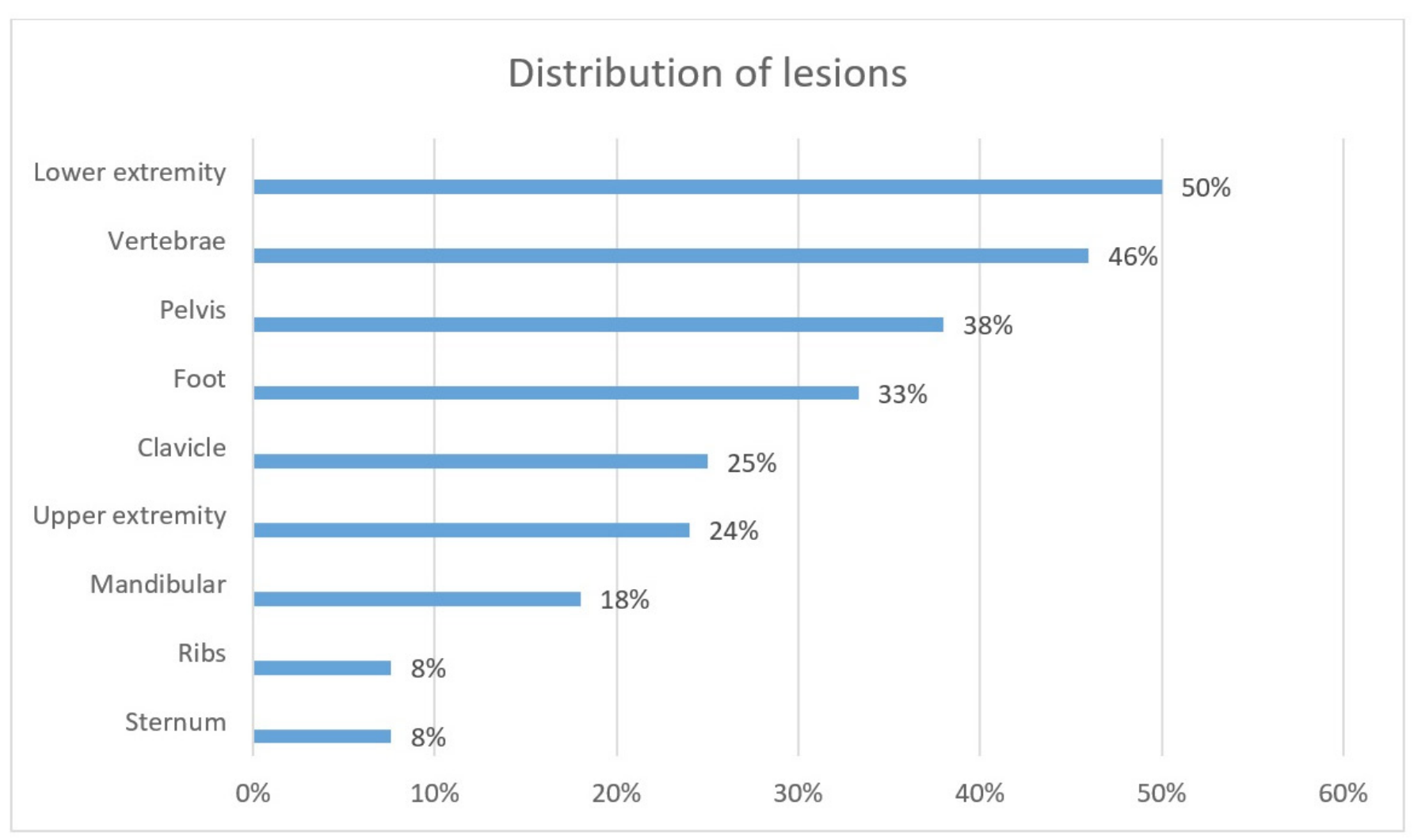

Figure 3 Distribution of lesions in chronic non-bacterial osteitis.

was reported in $53 \%$ of cases. Trauma encompassed both physical traumas, such as a fall $(\mathrm{n}=11,20 \%)$, and psychological traumas. Physical traumas $(n=18,32 \%)$ included not only falls but also dog bites, fractures, intravenous needles and others. Psychological traumas $(\mathrm{n}=7,13 \%)$ comprised bullying, and familial and school problems.

\section{Number of lesions and localisations}

At first manifestation, 20\% reported one lesion, 50\% reported two to five lesions and $27 \%$ reported more than five lesions. During the course of disease, further lesions were confirmed in $51 \%$ of CNO cases, with $21 \%$ being located within 6 months from initial diagnosis. The distribution of lesions can be found in figure 3. Most lesions were in the metaphyses of long bones, pelvis, lower extremities and feet. Vertebral lesions were found in $30 \%$ of cases in the first step of diagnosis. In $30 \%$ of cases, the patients' chief complaint was back pain, which led to further diagnostics focusing on the vertebrae. Approximately $11 \%$ already had a vertebrae plana at first diagnosis. Further lesions in the spinal column were diagnosed during the course of the disease in $18 \%$ of patients without initial vertebral lesions; lesions in the cervical spine were reported in $16 \%$ of patients, in the thoracic spine $28 \%$, in the lumbar spine $18 \%$ and in the sacrum and coccyx $18 \%$.

Circa $20 \%$ of patients reported a unifocal lesion.

\section{Treatment}

Differing initial diagnoses (bone malignancies) resulted in three patients receiving chemotherapy for approximately 12 months.

Non-steroidal anti-inflammatory drugs (NSAIDs) such as ibuprofen (61\%), naproxen (50\%), indometacin $(23 \%)$ and diclofenac $(20 \%)$ were prescribed in $95 \%$ of all patients, and NSAIDs and steroids $(33 \%)$ were the most commonly prescribed therapy after the CNO diagnosis. Forty-six per cent of all patients answered the question, what NSAID provided the best relief of symptoms. Sixty-five per cent of this group reported naproxen as the NSAID with the most beneficial impact and ibuprofen at $35 \%$ as the second most beneficial.

Although NSAIDs and steroids were the most commonly prescribed drugs for $\mathrm{CNO}$, bisphosphonates and biologics were frequently used in patients with severe courses of disease. Bisphosphonates made up 21\% ( $n=22)$ of the therapeutic agents, with pamidronate $(n=18)$ as the most commonly prescribed. From the 22 patients who were receiving a bisphosphonate, $68 \%(\mathrm{n}=15)$ had vertebral lesions. Over $14 \%$ of patients received a biologic agent: $9.5 \%$ etanercept, $2 \%$ adalimumab, $2 \%$ infliximab and $1 \%$ golimumab. Of the $14 \%$ of patients which received biologics, $7 / 15$ had lesions on the pelvis, $7 / 15$ on the clavicle, $5 / 15$ in the mandible and 5/15 on the spinal column. Most of these patients had multiple lesions, with one patient being affected throughout the entire spinal column (cervical, thoracic and lumbar), clavicle, pelvis and feet. 


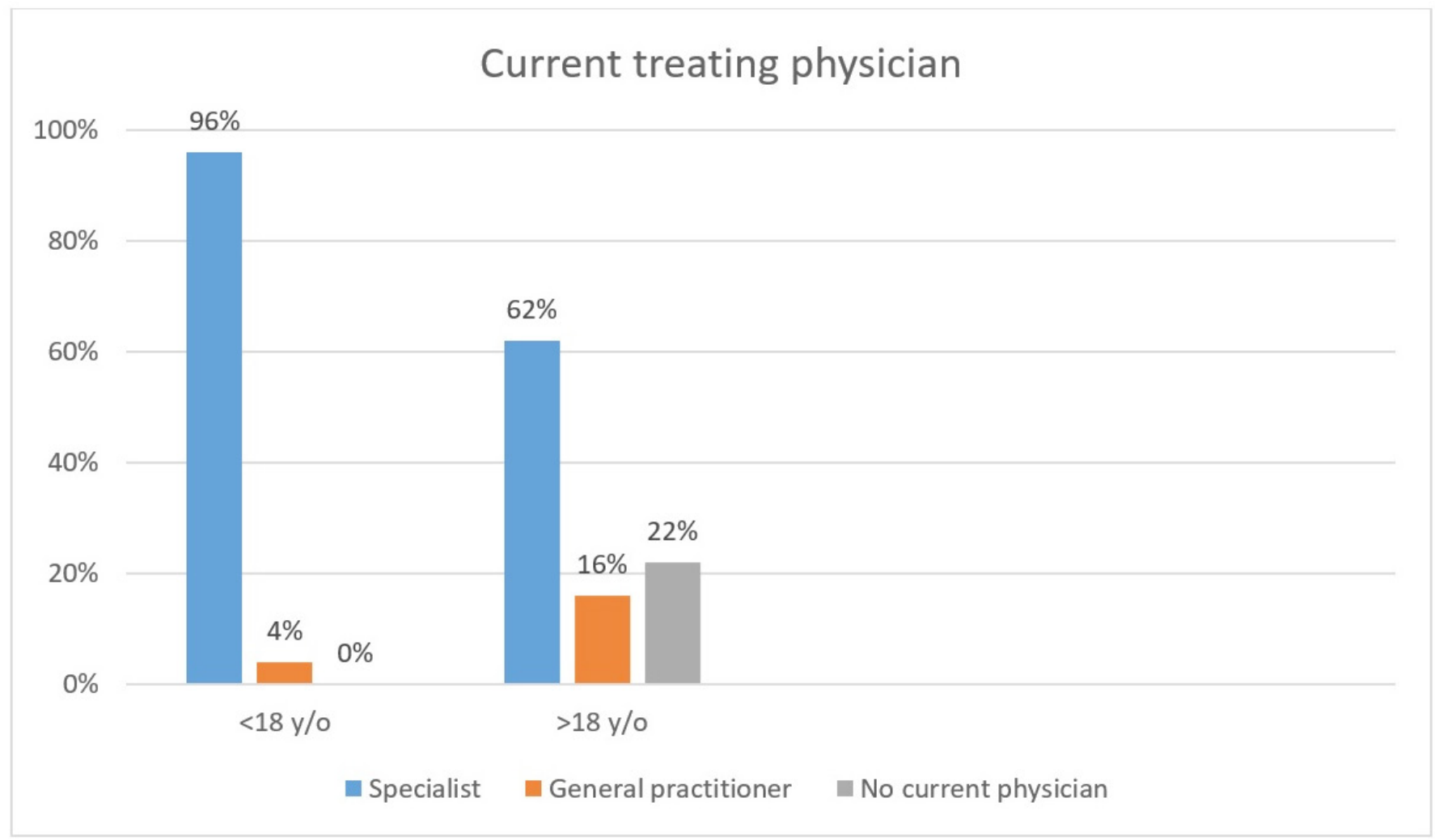

Figure 4 Current treating physician for chronic non-bacterial osteitis.

\section{Associated diseases}

CNO-related diseases were present in 28\% (30/105) of this patient population. Of the associated diseases, skin disorders dominated with $67 \%$ including palmoplantar pustulosis $(9 / 30)$, psoriasis $(5 / 30)$ and severe acne $(6 / 30)$. Other reported associated diseases included arthritis (9/30; 33\% adjacent to lesion) and Crohn's disease $(n=1)$.

Associated diseases in family members were revealed in $16 / 105(15 \%)$ fathers and 16/105 (15\%) mothers. Again, the skin lesions such as psoriasis $(34 \%)$ and palmoplantar pustulosis (13\%) were predominant. Other rheumatic diseases like chronic polyarthritis were reported in 10/16 females and 3/16 males. Crohn's disease (2/16) and ulcerative colitis $(1 / 16)$ were diagnosed in fathers of our patients.

\section{Patient care}

From the paediatric population, $96 \%$ were being treated by a paediatric rheumatologist or an orthopaedic surgeon, whereas with the adult population only $62 \%$ were being treated by a specialist (defined by a rheumatologist or an orthopaedic surgeon) and $16 \%$ by a general practitioner (figure 4.) From the 32 patients $>18$ years old, $22 \%$ had no treating physician; from these patients with no treating physician, $4 / 7$ no longer had an active disease at time of survey and $7 / 7$ patients were between the ages of 18 and 28 years.

The distance to the treating physician varied widely; however, $45 \%$ had to travel $25 \mathrm{~km}$ or less and $86 \%$ travelled $100 \mathrm{~km}$ or less, and one patient travelled up to $300 \mathrm{~km}$ to a specialist. Patients were asked how well cared for do they feel from their specialists, and on a VAS from 1 to $10,>50 \%$ responded with an 8 or higher. Patients were often referred to or specifically asked for a referral to see a physical therapist in $64 \%$ of cases.

CNO had reported negative effects in $44 \%$ of cases on the entire family, with another $39 \%$ reporting a partial effect on the family. CNO affected close family members, and friendships, school and work life. From patients which reported difficulty in friendships, $56 \%$ described, at minimum, a partial negative effect on relationships. In comparison, however, due to this disorder, $79 \%$ reported that $\mathrm{CNO}$ has negatively affected either school or work.

Seventy-five per cent of all patients received no type of psychosocial guidance, although $49 \%$ would have liked to have consultation with a guidance counsellor or psychologist. These numbers correlate with the $51 \%$ of patients and family members which felt uninformed regarding the NBO diagnosis and the course of disease.

Periods of absences from school or work did not vary widely between before the diagnosis and afterwards. The largest change in the number of days absent per year due to $\mathrm{CNO}$ was in the 6-20 day category; before the diagnosis, patients reported absences at $22 \%$ and afterwards at $31 \%$. However, absences greater than 20 days saw a $5 \%$ drop after the diagnosis, from $30 \%$ to $25 \%$.

Patients were also questioned as to what they would most like to learn and hear about at the information 


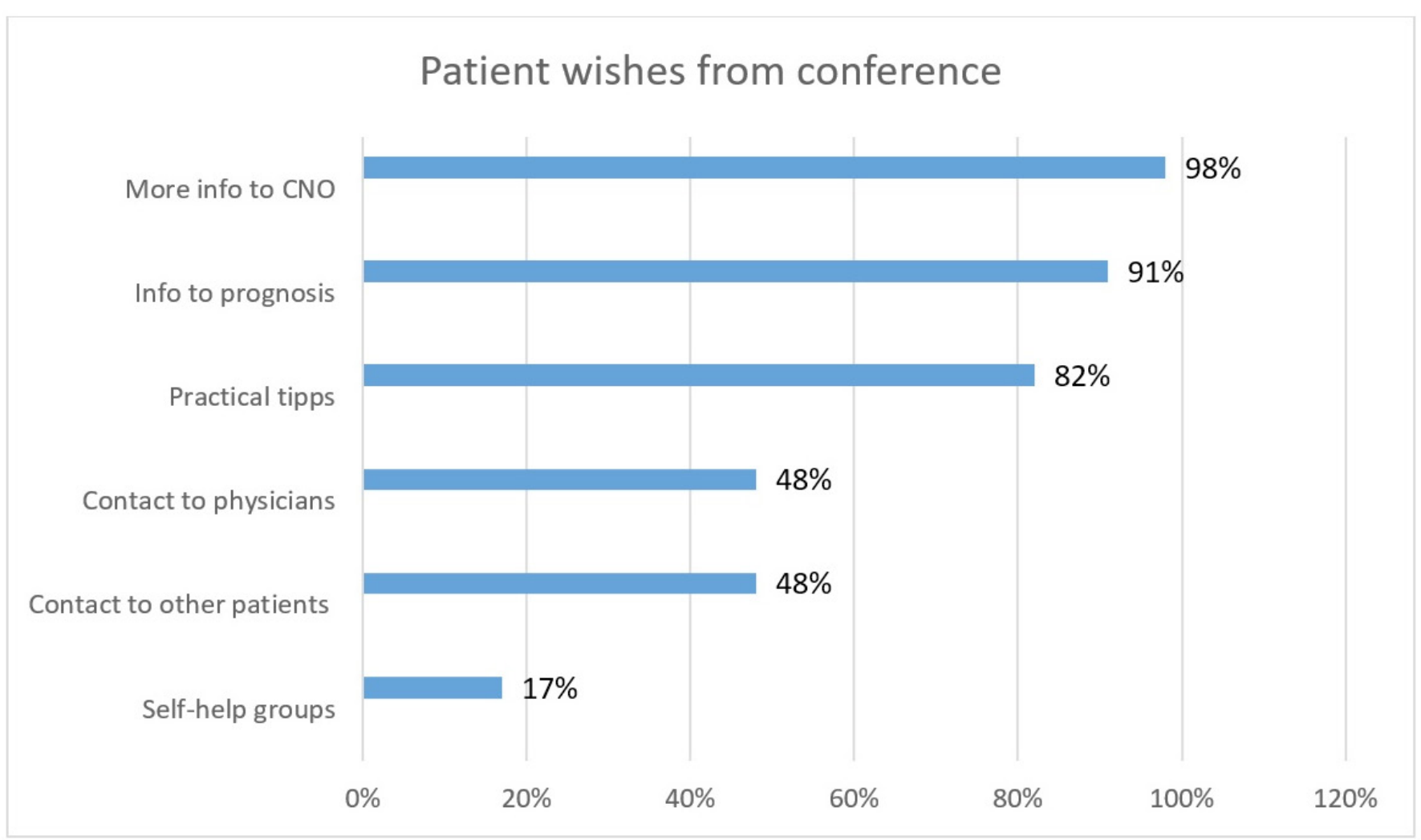

Figure 5 Patient wishes from CNO conference. CNO, chronic non-bacterial osteitis; info, information; NBO, non-bacterial osteitis.

day: specifically-more general information to $\mathrm{CNO}$, information to prognosis, practical tips, contact to physicians with CNO expertise, contact to other patients and building of self-help groups (figure 5.)

Other topics of interest ranged from typical side effects of medications to pregnancy to nutrition and alternative therapy options. Many of the paediatric patients and family members were concerned with the transition into adulthood and what effect $\mathrm{CNO}$ would have later in life.

\section{DISCUSSION}

To our knowledge, this is the first health services research to assess diagnosing, treatment and the psychosocial aspect of CNO from a patient perspective with such a large population pool.

\section{Medical data}

Overall, the patient derived information concerning their own illness matches the current medical literature. The number of lesions, localisation of lesions, therapy plan, inflammation parameters and imagery used (data not shown) is comparable to previous scientific literature. $^{613-17}$ This leads to the conclusion that the group of patients in attendance on the two conference days were well informed, have read about $\mathrm{CNO}$ and were seeking further information.

\section{Delay of diagnosis}

Patients reported long lag times from the onset of symptoms until diagnosis. Approximately $70 \%$ of the patients were diagnosed within 18 months from the onset of symptoms, but still $7 \%$ had to wait more than 5 years. These lag times lead to patient stress, both physical and emotional, and unnecessary testing and treatment. Delays in the diagnosis may lead to prolonged use of antibiotics, multiple surgeries, repeated bone biopsies and excessive radiation exposure. Another contributing factor to the long lag times in diagnoses and treatment is the distance to specialists. In Germany, most paediatric rheumatologists are located in larger cities and at university hospitals, and adult rheumatologists often have long wait lists. Therefore, patients often resort to being treated either by a general practitioner or a paediatrician.

Circa $20 \%$ of patients reported a unifocal lesion. However, from the 21 patients reporting 1 lesion, only 5 (24\%) received a whole-body MRI and $6(29 \%)$ a bone scan. This often led to a different differential diagnosis, mostly bacterial osteomyelitis, and a different therapy plan. This resulted in another delay in diagnosis.

\section{Therapy}

With $27 \%$ continuing with antibiotic therapy after diagnosis, there must be still uncertainty in the medical community regarding the $\mathrm{CNO}$ diagnosis and the proper treatment plan once recognised. A stepwise guide for 
the therapeutic treatment of $\mathrm{CNO}$ was developed to alleviate pain and prevent further degeneration; the plan highlights the use of NSAIDs in the first-line treatment of CNO ${ }^{18}$ Currently, there are national and international efforts to establish validated treatment protocols for $\mathrm{CNO}$.

The long lag times in diagnosis and the continuation of antibiotic therapy among other factors lead to the conclusion that there is a need for better clarification and education regarding NBO.

\section{Psychosocial and socioeconomic aspects}

As with most chronically ill patients, absences from school and work are of great importance. These absences have an effect on school performance, promotions and the emotional well-being of the patient. When comparing the number of absences before and after the diagnosis, there is very little difference. Which leads to the questions, is the medical therapy successful or does pain amplification play a significant role in the patient group in attendance at the conference? However, according to the patients, most had seen a significant pain level drop when comparing onset to current conditions, with most patients starting with a median pain level of $8 / 10$ (IQR: 6.5-9) and dropping to 4/10 (IQR: $1.5-6$ ) after treatment. On the other hand, pharmacological therapy and psychosocial aspects have a great influence on well-being and quality of life. Three-quarters of all patients did not receive psychosocial support. Half of all patients would have liked to have consultation with a guidance counsellor or psychologist.

More than $80 \%$ reported that CNO has had a negative influence on family life. Physicians caring for chronically ill patients should be aware how this illness affects especially young patients, and other family members and members of the support structure. In Germany, unfortunately, interdisciplinary care can only be offered in specialised medical centres.

\section{Transition and adult patients}

From the adult population in attendance, $22 \%$ were not seeing a specialist and had no treating physician for CNO. These patients vary in ages between 18 and 28 years old. This highlights the need for a better transition model from paediatric care to adult care, as all of these patients were diagnosed as children with CNO.

Especially, in Anglo-American countries, there are transition clinics where the needs of chronically ill young adults are met. ${ }^{19-21}$ In Germany, a transition model for patients with chronic rheumatic illnesses was developed. ${ }^{19} 22$ This model helps patients coordinate care transitioning from the paediatric community into the adult community and works together with both communities to assure a seamless transition. Once transition is complete, this is followed up to ascertain and highlight any needs for improvement. Although such models exist in Germany, this transition care is not widespread, and leaves many patients without a healthcare provider for chronic illnesses after the age of 18 .
A large portion of the study's population felt uninformed regarding this illness. This was the top reason for visiting the conference; patients needed and wanted more information about CNO (98\%). Practical tips and information to prognosis were also important topics. With such small percentages of patients with $\mathrm{CNO}$, attendance at our conference represented the thirst for information that these chronically ill patients have.

\section{CONCLUSION}

To our knowledge, this is the first study highlighting the impact of CNO from the patient perspective. Delay of diagnosis, living with differential diagnoses like malignancies and finding specialists for medical care drive patients to search for more information. Interested patients were able to report their disease precisely, so that patient data matched medical literature concerning $\mathrm{CNO}$ very well. Nevertheless, this survey shows very clearly that psychosocial and socioeconomic aspects need to be addressed. Negative impact on family, work and friendships seems to influence partaking in daily life. Support is especially necessary in adolescents and young adults, who often dropped out of medical attendance.

For the incidence rate of this disease, $0.45 / 100000,{ }^{13}$ 105 patients is large but a relative snapshot in time. Therefore, prospective evaluations of independent patient populations would give more insight.

\section{Limitations}

As with most health services research, patient subjectivity remains to be a problem. Some of the surveys were either not completely filled in or answers were given that did not match the question which often led to the participant's answer being disregarded. In an attempt to restrain the time and burden on patients, the questionnaire was kept short, therefore limiting the information which could be collected. Often patients were diagnosed years previously with $\mathrm{CNO}$, and neither the patient nor the parents could recall initial symptoms or pain levels. In attendance were typically patients with a more severe course of disease and that were very well informed about this disease. This could also explain why the patients' data were very comparable with previous research.

Contributors AFJ developed the idea for the study. CCGS and AFJ were involved in the study conception, preliminary literature review and design of the search strategy and the study protocol. SG and JG designed the initial database to record study inputs and completed initial data input. CCGS concluded data input and drafted the report, which was critically reviewed and approved by all authors. CCGS and VG were involved in screening and data extraction of papers. CCGS, AFJ and VG reviewed data extraction output.

Funding The study was funded by the non-profit organisations Kinder-Rheumahilfe Muenchen e.V., Edith-Haberland-Wagner-Stiftung and Kindness for Kids, Munich. Through financial pledges, all three organisations provided the necessary capital for the two patient conferences. In addition, Kinder-Rheumahilfe Muenchen also organised the two conferences. The study itself and the work thereafter were unfunded.

Competing interests None declared. 
Patient consent Detail has been removed from this case description/these case descriptions to ensure anonymity. The editors and reviewers have seen the detailed information available and are satisfied that the information backs up the case the authors are making.

Ethics approval Ethics committee of the medical faculty at Ludwig-Maximilian University (Munich).

Provenance and peer review Not commissioned; externally peer reviewed.

Data sharing statement All available data can be obtained by contacting the corresponding author.

Open Access This is an Open Access article distributed in accordance with the Creative Commons Attribution Non Commercial (CC BY-NC 4.0) license, which permits others to distribute, remix, adapt, build upon this work non-commercially, and license their derivative works on different terms, provided the original work is properly cited and the use is non-commercial. See: http://creativecommons.org/ licenses/by-nc/4.0/

(C) Article author(s) (or their employer(s) unless otherwise stated in the text of the article) 2017. All rights reserved. No commercial use is permitted unless otherwise expressly granted.

\section{REFERENCES}

1. Hayem G, Bouchaud-Chabot A, Benali K, et al. SAPHO syndrome: a long-term follow-up study of 120 cases. Semin Arthritis Rheum 1999;29:159-71.

2. Jansson $A F$, Müller TH, Gliera L, et al. Clinical score for nonbacterial osteitis in children and adults. Arthritis Rheum 2009;60:1152-9.

3. Ferguson PJ, El-Shanti HI. Autoinflammatory bone disorders. Curr Opin Rheumatol 2007;19:492-8.

4. Girschick HJ, Zimmer C, Klaus G, et al. Chronic recurrent multifocal osteomyelitis: what is it and how should it be treated? Nat Clin Pract Rheumatol 2007;3:733-8.

5. El-Shanti HI, Ferguson PJ. Chronic recurrent multifocal osteomyelitis: a concise review and genetic update. Clin Orthop Relat Res 2007;462:11-19.

6. Jansson A, Renner ED, Ramser J, et al. Classification of nonbacterial osteitis: retrospective study of clinical, immunological and genetic aspects in 89 patients. Rheumatology 2007;46:154-60.

7. King SM, Laxer RM, Manson D, et al. Chronic recurrent multifocal osteomyelitis: a noninfectious inflammatory process. Pediatr Infect Dis J 1987;6:907-11.
8. Kahn MF. [Current status of the SAPHO syndrome]. Presse Med 1995;24:338-40.

9. Anderson SE, Heini P, Sauvain MJ, et al. Imaging of chronic recurrent multifocal osteomyelitis of childhood first presenting with isolated primary spinal involvement. Skeletal Radiol 2003;32:328-36.

10. Maslow GR, Haydon A, McRee AL, et al. Growing up with a chronic illness: social success, educational/vocational distress. J Adolesc Health 2011;49:206-12.

11. Shapiro J. Family reactions and coping strategies in response to the physically ill or handicapped child: a review. Soc Sci Med 1983;17:913-31.

12. Northam EA. Psychosocial impact of chronic illness in children. J Paediatr Child Health 1997;33:369-72.

13. Grote V, Silier CC, Voit AM, et al. Bacterial osteomyelitis or nonbacterial osteitis in children: a study involving the German surveillance unit for rare diseases in childhood. Pediatr Infect Dis $J$ 2016. doi: 10.1097/INF.0000000000001469 [Epub ahead of print 13 Dec 2016].

14. Peltola $\mathrm{H}$, Pääkkönen $M$, Kallio $P$, et al. Short- versus long-term antimicrobial treatment for acute hematogenous osteomyelitis of childhood: prospective, randomized trial on 131 culture-positive cases. Pediatr Infect Dis J 2010;29:1123-8.

15. Jansson A, Grote V. Non-bacterial osteitis and bacterial osteomyelitis. Monatsschrift Kinderheilkunde 2009;157:718-9.

16. Copley LA, Kinsler MA, Gheen T, et al. The impact of evidencebased clinical practice guidelines applied by a multidisciplinary team for the care of children with osteomyelitis. J Bone Joint Surg Am 2013:95:686-93.

17. Jorge LS, Chueire AG, Rossit AR. Osteomyelitis: a current challenge. Braz J Infect Dis 2010;14:310-5.

18. Jansson AF, Borte M, Hospach A, et al. Diagnostics and therapy of non-bacterial osteitis. Consensus statement of the 16th Worlitz expert meeting 2013 for the Deutsche Gesellschaft fur Kind- und Jugendrheumatologie. Monatsschrift Kinderheilkunde 2014;162:539-45.

19. Foster HE, Minden K, Clemente D, et al. EULAR/PReS standards and recommendations for the transitional care of young people with juvenile-onset rheumatic diseases. Ann Rheum Dis 2017;76:639-46.

20. McDonagh JE. Transition of care from paediatric to adult rheumatology. Arch Dis Child 2007;92:802-7.

21. McDonagh JE, Southwood TR, Shaw KL. The impact of a coordinated transitional care programme on adolescents with juvenile idiopathic arthritis. Rheumatology 2007:46:161-8.

22. Schalm S, Jansson AF. [Transitional care in rheumatology in Germany]. Internist 2012;53:1054-62. 